\title{
Inverse modeling of indoor instantaneous airborne contaminant source location with adjoint probability-based method under dynamic airflow field
}

\author{
Haidong Wang ${ }^{1, *}$, Sai Lu ${ }^{1}$, Jiajia Cheng ${ }^{1}$, Zhiqiang (John) Zhai ${ }^{2}$
}

${ }^{1}$ School of Environment and Architecture, University of Shanghai for Science and Technology. 516 Jungong Road, Shanghai, 200093. P.R.C

${ }^{2}$ Department of Civil, Environmental and Architectural Engineering, University of Colorado Boulder. 428UCB, Boulder, 80309-0428, Colorado, USA.

*Corresponding email: whd@usst.edu.cn

\begin{abstract}
Accurate and prompt identification of airborne contaminant source location in indoor environment is of vital importance for building operation safety. Successful inverse tracking algorithm to identify airborne contaminant location usually requires limited sensor readings and indoor airflow information as input. Such mathematical algorithm under steady-state indoor airflow scenario has been intensively investigated. However, in many built environment scenarios, air velocity direction and magnitude keeps changing with time, making the transportation process of airborne contaminant significantly different from it under steady-state airflow. This paper mainly focuses on the airborne contaminant source location identification under dynamic airflow, by employing an adjoint probability-based inverse tracking method. The mathematical model and process of indoor instantaneous contaminants source location identification under dynamic air flow field is investigated and presented. Case studies using Computational Fluid Dynamics (CFD) tool by unsteady RANS simulation are conducted on a subway station model as well as an aircraft cabin, in which case experimental validation is also conducted. The capability of the new method is verified for air contaminant source location identification under dynamic indoor airflow.
\end{abstract}

\section{PRACTICAL IMPLICATIONS}


Successful identification of airborne contaminant source location dynamic airflow field implies the capability of the method to locate an airborne contaminant source by limited sensor information and time-dependent airflow field in real-time. Practically the timedependent airflow field data can be obtained through CFD simulation with unsteady RANS model and monitored time-dependent boundary condition.

\section{KEYWORDS}

Dynamic airflow, inverse modeling, adjoint probability method, Conditioned Adjoint Location Probability $(C A L P)$, indoor airborne contamination, location identification

\section{INTRODUCTION}

Indoor airborne contaminant received great attention recent years for indoor environment quality (IEQ) concern and building safety issue. When a certain concentration of toxic airborne contaminant was released into enclosed spaces, the contamination will quickly spread to the entire space and cause serious consequences. Therefore, prompt and accurate identification of airborne contaminant source characteristics will greatly assist isolation and removal of the source, therefore diminishes the consequence of such incidence effectively. Such process of identifying the source releasing location and possibly releasing time by limited sensor reading is a typical inverse problem.

Inverse problems are common and widely investigated in various applications, such as inverse modeling of the atmospheric pollution to identify the location[1,2,3,4] and releasing rate[5, 6$]$ of air pollutant source, diffusion of carbon monoxide concentrations in forest fires to deal with sudden accidents[7], and design of aircraft cabin environment[8]. In typical built environment, airborne contaminant transports with airflow through convection and diffusion, therefore airflow field information is indispensable part of input data for such algorithms. A sensor system providing concentration readings is another input for the model. Algorithms 
and case studies of identifying indoor airborne contaminant have been well established under different scenarios following such ideology.

Zhai categorized inverse tracking problem to three main types[9], which are forward method $[10,11,12]$, reverse method[9,13,14], and probability calculation method[15]. The forward method usually requires large amount of computation to trial all possible source and its consequences and therefore time-consuming. It diminishes the role of inverse modeling in the real-time identification of source location. To overcome such disadvantages, Sohn used Bayesian statistics to interpret the pollutant concentrations from several sensors which were installed in the building, and then computed the best uncertainties conditions of the pollutant source[16]. Bayesian probability theory was employed to identify the gaseous pollutant source by interpreting real-time monitoring information[17,18]. An artificial neural network based method working with multi-zone model is an alternative for speed purpose[19,20]. Cai and $\mathrm{Li}[21,22$,]conducted pollutants real-time positioning of pollutants releasing at a constant speed and multiple sources by a linear programming model built on an analytical expression of indoor contaminant dispersion.

Two typical backward inverse methods named quasi-reversibility(QR) and pseudoreversibility(PR) were proposed to study the localization of gaseous[23] and particulates[24] pollutants released by steady and unsteady pollutant source in an enclosed cabin[25]. Zhang extended QR to Lagrangian-reversibility(LR) method[26] for particle tracking and improved the accuracy of the backward inverse method. Besides, Zhang also employed an inverse method based on Tikhonov regularization and least-squares optimization to calculate the mass of the pollutant[27] and then proposed an inverse matrix submodel to inversely determine the temporal release rate profile and a Bayesian probability submodel to identify its releasing location and the corresponding sensor alarming time[28]. 
Liu and Zhai used probability algorithm to identify location of the instantaneous[29,30] and dynamic[29] indoor contaminant source, and such probability-based inverse multi-zone model was utilized to successfully track contaminant source location with known source releasing time in a multi-zonal building. This method is proved to be successful in assisting the design of a high-performance sensors network system and identifying contaminant source locations for an entire building based on limited sensors[15].

Most, if not all, of the previous studies mentioned assumed steady-state indoor/outdoor airflow field during releasing of airborne contaminant source. Such airflow field information, obtained by measurement or through computer modeling, is one of the key input parameters for these problems. In the real case scenarios, however, air velocity and temperature may change at any time in instances such as variable air volume system (VAV) and natural ventilation environment. In certain special cases airflow field changes periodically with time. This imposes a great challenge for contaminant source identification method of previous studies.

In this paper, a mathematical model and process for identifying an instantaneous airborne contaminant location under dynamic airflow indoor environment is developed and investigated based on the well-established adjoint probability method. The indoor airflow field information obtained from time-dependent computational fluid dynamics (CFD) simulation is utilized. Limited sensor readings are used as input parameter. Case studies with typical dynamic airflow conditions verify the capability of such model and process in application of time-dependent/dynamic airflow field circumstance.

\section{METHODS}




\subsection{Principles of probability-based inverse modeling method}

Probability method of inverse modeling is used to estimate the probability of an event as a target probabilistic function, through the maximum or minimum and the objective function to find out the information of the source. Neupauer and Wilson[31, 32] developed a method to predict water pollutant source location and travel time probabilities in the earlier years. Because of the similar spreading characteristics of pollutants in water and air, Liu and Zhai[9] extended this method and applied it to indoor environment to identify the potential indoor pollutant source characteristics with limited sensor readings. This method has been intensively validated for steady-state airflow field scenario.

With a focus on unsteady airflow field, in which instantaneous indoor air contaminant sources location is identified, this paper extends the adjoint probability method for the application of time-dependent airflow field. The basic principle and the corresponding probability equations of the adjoint probability method based on CFD are introduced below.

\subsection{Fundamentals of adjoint probability method under dynamic airflow}

Assume that there is an instantaneous point contaminant source of a total mass $M_{0}$ in an enclosed environment at certain position of $\vec{x}=\vec{x}_{0}$, which releases the contaminant into the air at time $t=0$. The contaminant will spread to the entire space in a finite time. At $t=T>0$, any location in the enclosed environment can be considered as the potential source of contamination as long as the contaminant through the air flow reaches it, and the probability of the potential contaminant source is defined as the forward location probability[33,34,35]. For example, the contaminant source though the air flow spread to the finite volume $\Delta V_{1}$ at the position of $\vec{x}=\vec{x}_{1}$ is $M_{1}$ when $t=T$, the forward location probability at the position of $\vec{x}=\vec{x}_{1}$ at time $t=T$ can be expressed as Eq. (1). 


$$
\mathrm{P}\left(\Delta V_{1} \mid \vec{x}=\vec{x}_{1}, t=T, \vec{x}_{0}\right)=\frac{M_{1}}{M_{0}}
$$

Using $f_{x}$ to represent the per volume probability value, this equation can be expressed as:

$$
f_{x}\left(\vec{x}, t=T, \vec{x}_{0}\right)=\frac{P\left(\Delta V_{1} \mid \vec{x}=\vec{x}_{1}, t=T, \vec{x}_{0}\right)}{\Delta V_{1}}=\frac{M_{1}}{M_{0}} / \Delta V_{1}=\frac{C_{1}}{M_{0}}
$$

In equation (2), $C_{1}$ represents the concentration value of the contaminant at $\vec{x}=\vec{x}_{1}$. A general format of Eq. (2) is:

$$
f_{x}\left(\vec{x}, t=T, \vec{x}_{0}\right)=\frac{C(\vec{x}, T)}{M_{0}}
$$

In equation (3), $f_{x}\left(\vec{x}, t=T, \vec{x}_{0}\right)$ represents the probability that certain position being the potential contaminant source at $t=T, \vec{x}$ is random position in the enclosed environment, $C(\vec{x}, T)$ represents the concentration value of contaminant at $t=T$ due to the release of the instantaneous point contaminant source. The right side of Equation (3) is the ratio of such concentration to the total released mass at position of $\vec{x}_{0}$, therefore can be generalized as $\frac{d C(\vec{x}, T)}{d M_{0}}$. Using $\psi_{x}\left(\vec{x}, t=T, \vec{x}_{0}\right)$ to represent such ratio will lead to:

$$
f_{x}\left(\vec{x}, t=T, \vec{x}_{0}\right)=\frac{d C(\vec{x}, T)}{d M_{0}}=\psi_{x}\left(\vec{x}, t=T, \vec{x}_{0}\right)
$$

The forward location probability density function $f_{x}\left(\vec{x}, t=T, \vec{x}_{0}\right)$ can be interpreted as the probability of an instantaneous point contaminant source at the location of $\vec{x}_{0}$, spreading to reach location $\vec{x}$ after a fixed period of time $t=T$. Define $\tau=T$ - $t$ as backward time to reversely perceive this contaminant spreading process, forward location probability can be expressed as $f_{x}\left(\vec{x}=\vec{x}_{0}, \tau=T, \vec{x}\right)$ and $\psi_{x}\left(\vec{x}_{0}, \tau=T, \vec{x}\right)$. Use $\psi_{x}{ }^{*}\left(\vec{x}_{0}, \tau=T, \vec{x}\right)$ to differentiate it from the forward probability, it can be written as:

$$
f_{x}\left(\vec{x}=\vec{x}_{0}, \tau=T, \vec{x}\right)=\psi_{x}^{*}\left(\vec{x}_{0}, \tau=T, \vec{x}\right)
$$

In Eq. (5), $\psi_{x} *\left(\vec{x}_{0} ; \tau=T, \vec{x}\right)$ is defined as backward location probability[9], indicating the degree of possibility to which a contaminant source is at a certain position. Calculating the contaminant transportation is a significant part of this process, and simulating of certain 
species transport usually adopts CFD method in practice. The general format of governing equations that CFD solves is given as following:

$$
\frac{\partial \Phi}{\partial t}+\nabla(\vec{V} \cdot \emptyset)-\Gamma_{\phi} \nabla^{2} \emptyset=S_{\varnothing}
$$

In equation (6), $t$ is time, $\Phi$ is a generic variable that can be used to represent different variables, such as velocity, temperature and concentration. For each variable $\Phi, \Gamma_{\phi}$ is the corresponding diffusion coefficient, and $S_{\emptyset}$ is source term. A series of air velocity field data can be obtained at the end of each time steps as $V_{j}=\left(V_{j}^{1}, V_{j}^{2}, \cdots V_{j}^{i} \cdots V_{j}^{n-1}, V_{j}^{n}\right)$ through solution of equation (6). This is the CFD governing equation thus solution of this equation can be implemented by any commercial CFD software. In this research, commercial CFD software Phoenics was used. The mass of the contaminants transfer equation and boundary conditions are as follows:

$$
\begin{aligned}
& \frac{C^{1}-C^{0}}{\Delta t}+\frac{\partial V_{j}^{1} C^{1}}{\partial x_{j}}=\frac{\partial}{\partial x_{j}}\left[v_{c} \frac{\partial C^{1}}{\partial x_{j}}\right]+\left(S_{c}+q_{1} C_{I}^{1}-q_{0} C^{1}\right) \\
& \frac{C^{2}-C^{1}}{\Delta t}+\frac{\partial V_{j}^{2} C^{2}}{\partial x_{j}}=\frac{\partial}{\partial x_{j}}\left[v_{c} \frac{\partial C^{2}}{\partial x_{j}}\right]+\left(S_{c}+q_{1} C_{I}^{2}-q_{0} C^{2}\right)
\end{aligned}
$$

$\vdots$

$$
\frac{C^{n}-C^{n-1}}{\Delta t}+\frac{\partial V_{j}^{n} C^{n}}{\partial x_{j}}=\frac{\partial}{\partial x_{j}}\left[v_{c} \frac{\partial C^{n}}{\partial x_{j}}\right]+\left(S_{c}+q_{1} C_{I}^{n}-q_{0} C^{n}\right)
$$

$C(\vec{x}, 0)=C^{0}(x)$

$C^{i}(\vec{x}, t)=g_{1}(t)$ on $\Gamma_{1}$

$\left[v_{c} \frac{\partial C^{i}}{\partial x_{j}}\right] n_{i}=g_{2}(t)$ on $\Gamma_{2}$

$\left[V_{j}^{i} C^{i}-v_{c} \frac{\partial C^{i}}{\partial x_{j}}\right] n_{i}=g_{3}(t)$ on $\Gamma_{3}$

In equation (7), $\rho$ represents the density of air, $C^{i}$ the concentration of contaminants of the $i^{\text {th }}$ measurement, $V_{j}$ is the air velocity at $x_{j}$ direction of the $n^{\text {th }}$ time step under dynamic airflow scenario. Under dynamic airflow, it will change with time. $v_{c}$ is the effective turbulent diffusion coefficient of contaminant, $q_{0}$ is the per unit volume flow of contaminant outflow, 
$q_{1}$ is the per unit volume flow of contaminant inflow, $C_{I}{ }^{i}$ is the volume concentration of the contaminant corresponding to the $i^{\text {th }}$ time step, $S_{C}$ is all other forms of contaminant, therefore $S_{c}+q_{1} C_{I}{ }^{i}-q_{0} C^{i}$ is the sum of all external sources. $C^{0}$ is the initial concentration of each cell position, $V_{j}^{l}$ is the initial air velocity, $g_{1}, g_{2}$ and $g_{3}$ represent a known mathematic functions of the boundary condition of the contaminant. $\Gamma_{1}, \Gamma_{2}$ and $\Gamma_{3}$ are the three types of boundaries; $n_{i}$ is the outward unit normal vector in the $x_{i}$ direction. Most studies of contaminant transport usually set $q_{1}$ and $q_{0}$ to zero, because the increase or removal of contaminants is often associated with boundary conditions, depending on the inlet and exhaust. The solution of Eq. (7) requires obtained velocity field $V_{j}=\left(V_{j}^{1}, V_{j}^{2}, \cdots V_{j}^{i} \cdots V_{j}^{n-1}, V_{j}^{n}\right)$ obtained through previous step. First of all, contaminant source are released under the velocity field of $V_{j}^{1}$, only the contaminant concentration field will be solved at this time. $V_{j}^{1}$ was used in this step to advect contaminant source to get a new concentration field. After a time period of $\Delta t, V_{j}^{1}$ is replaced by $V_{j}^{2}$ to repeat this calculation. The contaminant concentration field information of each time step corresponding to $V_{j}=\left(V_{j}^{1}, V_{j}^{2}, \cdots V_{j}^{i} \cdots V_{j}^{n-1}, V_{j}^{n}\right)$ can be obtained.

This paper deals with backward location probability function density to predict the most probable source position in an indoor air contaminant event. Following such concept, Liu and Zhai[9] derived mathematical procedures for a certain fixed airflow field for the contaminant source location probability by utilizing the sensitivity analysis approach of Sykes et al[36]. In this paper, it is extended for a series of consecutive multiple airflow fields hereby for dynamically changed airflow field as follow:

$$
\begin{aligned}
& \frac{\Psi^{*, 1}-\Psi^{*, 0}}{\Delta \tau}-\frac{\partial V_{j}^{n} \Psi^{*, 1}}{\partial x_{j}}=\frac{\partial}{\partial x_{j}}\left[v_{c} \frac{\partial \Psi^{*, 1}}{\partial x_{j}}\right]+\left(-q_{0} \Psi^{*, 1}\right)+\frac{\partial h}{\partial C} \\
& \frac{\Psi^{*, 2}-\Psi^{*, 1}}{\Delta \tau}-\frac{\partial V_{j}^{n-1} \Psi^{*, 2}}{\partial x_{j}}=\frac{\partial}{\partial x_{j}}\left[v_{c} \frac{\partial \Psi^{*, 2}}{\partial x_{j}}\right]+\left(-q_{0} \Psi^{*, 2}\right)+\frac{\partial h}{\partial C}
\end{aligned}
$$




$$
\begin{aligned}
& \frac{\Psi^{*, n}-\Psi^{*, n-1}}{\Delta \tau}-\frac{\partial V_{j}^{1} \Psi^{*, n}}{\partial x_{j}}=\frac{\partial}{\partial x_{j}}\left[v_{c} \frac{\partial \Psi^{*, n}}{\partial x_{j}}\right]+\left(-q_{0} \Psi^{*, n}\right)+\frac{\partial h}{\partial C} \\
& \Psi^{*}(\vec{x}, 0)=\Psi^{*, 0}(\vec{x})=0 \\
& \Psi^{*}(\vec{x}, \tau)=0 \text { on } \Gamma_{1} \\
& {\left[v_{c} \frac{\partial \Psi^{*, i}}{\partial x_{j}}+V_{j}^{n+1-i} \Psi^{*, i}\right] n_{i}=0 \text { on } \Gamma_{2}} \\
& {\left[v_{c} \frac{\partial \Psi^{*, i}}{\partial x_{j}}\right] n_{i}=0 \text { on } \Gamma_{3}} \\
& \frac{\partial h}{\partial C}=\delta\left(\vec{x}-\vec{x}_{w}\right) \cdot \delta(\tau)
\end{aligned}
$$

In equation (8), $\Psi^{*, i}$ is the Standard adjoint location probability (SALP) of contaminant source calculated by $V_{j}^{n}$ through $V_{j}^{n+1-i}$, it can be source location or travel time probability of contaminant. $\tau$ is the backward time, $\vec{x}_{w}$ represents the position of the contaminant monitoring point, $\frac{\partial h}{\partial C}$ is the additional term of the source, and $\delta(x)$ is the impulse function. When $x=0, \delta(x)=1$, in other cases, $\delta(x)=0$. Compared with Eq. (7), the three types of boundary conditions of Eq. (8) have changed, the Dirichlet type boundary condition on $\Gamma_{1}$ keeps boundary type fixed; the Neumann type boundary condition on $\Gamma_{2}$ becomes Robin type; and the Robin-type boundary condition on $\Gamma_{3}$ becomes Neumann-type. $\Psi^{*}(\vec{x}, \tau=0)=$ 0 denotes the adjoint probability that all positions may be the potential source location is 0 at $\tau=0$. The adjoint probabilities are restrained by the boundary conditions at boundaries. The additional term of the source represents the probability that can be considered as potential source location at $\tau=0$. Under dynamic airflow scheme, information of airflow field of every time step, sensors location, and boundary condition of enclosed environment are needed to solve Eq. (8). For time-dependent problems, the airflow field information of every time step $\tau$ can be obtained by real-time measurement or unsteady CFD simulation. In Eq. (8), negative velocity value of $V_{j}^{n}$ in each time step is used. The general format of this equation is similar to regular CFD governing equation except this negative velocity. Therefore, before solving Eq. 
(8), direction of the velocity field of $V_{j}=\left(V_{j}^{1}, V_{j}^{2}, \cdots V_{j}^{i} \cdots V_{j}^{n-1}, V_{j}^{n}\right)$ which is obtained by solving the Eq. (6) will first be reversed by a Fortran code, and then the same method as used in Eq. (7) was employed to get the solution of $\Psi^{*, i}$ The forward and backward location probability for each sensor reading can be calculated by the solution of this equation.

\subsection{Joint probability for multiple alarming sensors}

Installing more sensors can refine the SALP calculation. During an incident, the alarm sensors record alarms instead of concentrations. Special treatments are needed to improve the accuracy of the location under this sensing scenario. The multiple-observation backward location probability, as suggested by Neupauer[37], can be calculated by combining multiple SALP simulation results of each sensor as Equation (9).

$f_{x}\left(X, \tau_{0}, X_{W_{1}}, X_{W_{2}}, \cdots X_{W_{N}}, \tau_{W_{1}}, \tau_{W_{2}} \cdots, \tau_{W_{N}}\right)=\frac{\prod_{k=1}^{N} f_{x}\left(X, \tau_{0}, X_{W_{k}}, \tau_{W_{k}}\right)}{\int_{x} \prod_{k=1}^{N} f_{x}\left(X, \tau_{0}, X_{W_{k}}, \tau_{W_{k}}\right) d x}$

Where $N$ represents the number of sensors, $X_{W_{k}}$ is the position of sensor-k $(k=1$ to $N), \tau_{W_{k}}$ is the time step size between different sensor measurements, and $\tau_{0}$ is the known reverse releasing time of the pollutant source as assumed in this paper.

\subsection{Joint probability equation for multiple sensor readings}

Installing more sensors will help improve location probability predictions by SALP calculation. In real case scenario, contaminant sensors have the ability to read and store historical concentration data. Lin[38] suggested a joint backward location probability for $\mathrm{N}$ sensor historical concentration measurements in order to improve the accuracy of the predicted position of the contaminant source, as follows:

$$
f_{x}\left(X \mid \hat{C}_{1}, \ldots, \hat{C}_{N} ; \tau_{0}, X_{1}, \ldots, X_{N}, \tau_{1}, \ldots, \tau_{N}\right)=\frac{\int_{M_{0}} \prod_{i=1}^{N} \mathrm{P}\left(\hat{C}_{i} \mid M_{0}, X ; \tau_{0}, X_{i}, \tau_{i}\right) f_{x}\left(X ; \tau_{0}, X_{i}, \tau_{i}\right) d M_{0}}{\int_{M_{0}} \int_{x} \mathrm{P}\left(\hat{C}_{i} \mid M_{0}, X ; \tau_{0}, X_{i}, \tau_{i}\right) f_{x}\left(X ; \tau_{0}, X_{i}, \tau_{i}\right) d x d M_{0}}
$$


Where $N$ represents the total number of measurements, $X_{i}$ is the location of sensor, $\tau_{i}=t-T$, is the measurement time (backward), and $\hat{C}_{i}$ is the contaminant concentration monitoring by the sensor at the backward time $\tau_{i} . \tau_{0}$ is the reverse release time of the pollution source, and it is known to us. $M_{0}$ is the assumed contaminant release intensity. $f_{x}\left(X ; \tau_{0}, X_{i}, \tau_{i}\right)$ is the SALP corresponding to the $i^{\text {th }}$ sensor reading. $P\left(\hat{C}_{i} \mid M_{0}, X ; \tau_{0}, X_{i}, \tau_{i}\right)$ is the probability distribution of the corresponding to the contaminant release intensity $M_{0}$ and source position $x$ from the $i^{\text {th }}$ concentration measurement. Neupauer and Wilson[39] suggested that $P\left(\hat{C} \mid M_{0}, X ; \tau_{0}, X_{i}, \tau_{i}\right)$ should be defined as a normal distribution as Eq. (11).

$\mathrm{P}\left(\hat{C} \mid M_{0}, X ; \tau_{0}, X_{i}, \tau_{i}\right) \sim N\left(M_{0} \cdot f_{x}\left(X ; \tau_{0}, X_{i}, \tau_{i}\right) \sigma_{\varepsilon}^{2}\right)$

In equation (11), $\hat{C}_{i}$ is corresponding to the possible contaminant concentration for the $i^{\text {th }}$ measurement by sensor, $\sigma_{\varepsilon}^{2}$ is the variance for the measurement error of the sensor. In this equation, we can combine multiple sensors of one-time reading or historical concentration readings from one sensor, as well as combine multiple sensors of historical concentration readings. To calculate the conditioned adjoint location probability (CALP), SALP should be obtained by solving Eq. (8). Figure 1 presents the main algorithm and procedure to predict the position of contaminant source by using the probability-based inverse CFD modeling method. The specific indoor environment case restrains the network of a sensor when it was first constituted. The dynamic air velocity field is measured or simulated by CFD tool in real time. The flow field data can be stored in computer as input for inverse modeling at any time that sensor detects contaminant concentration. The mathematical model introduced in this paper is then applied to identify the airborne contaminant source location. 


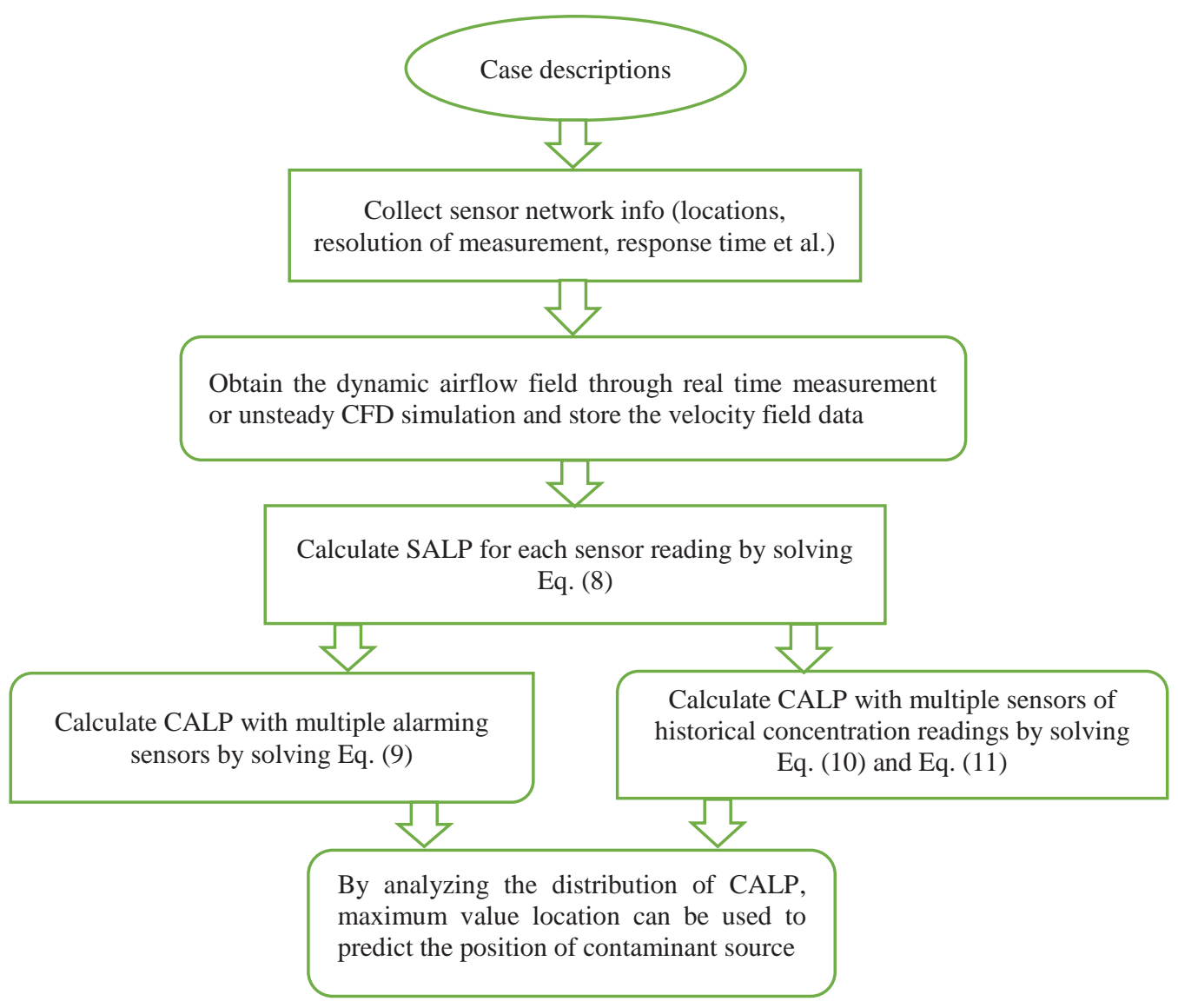

Figure 1. Procedure for predicting the position of contaminant source by using the probability-based inverse modeling method under dynamic airflow scenario

\section{RESULTS}

As the original adjoint probability method for identifying an instantaneous airborne contaminant source location under steady-state airflow in indoor environment is extended for dynamic airflow field, the applicability of the newly derived method is investigated by case studies. This was implemented by comparing the performance of the original and the new adjoint method. First, the accuracy of the original adjoint probability method for identifying an instantaneous airborne contaminant location under dynamic airflow was attempted; second, the accuracy of a newly developed mathematical model for identifying an instantaneous airborne contaminant location under dynamic airflow for the same scenario was validated. Two typical cases of dynamic airflow were selected for verification purpose. The first one is that when subway train passes through station and causes piston airflow effect, which is a 
typical circumstance that boundary condition changes with time and causes dynamic airflow. The second is the ventilation system in Boeing 737-200 airplane cabin environment, where hedging supply air results in periodically swinging airflow environment, which is a typical case that constant boundary condition causing periodically changing airflow field.

\subsection{Air contamination in subway station affected by moving train}

Subway station airflow is usually complex and dynamic, especially when train passes through and causes piston effect, which is a typical circumstance that dynamic airflow presents. Therefore, this study selected a scaled 2D subway station case to demonstrate the effectiveness of the algorithm for predicting the position of contaminant source. As shown in Figure 2 the plan view of the modeled subway station is $9 m$ long and $3 m$ wide, and isothermal condition was assumed so no energy equation was solved. In the station, air was supplied from the two slot inlets at side wall with a velocity of $1 \mathrm{~m} / \mathrm{s}$. A point contaminant source near the first inlet released instantaneously 100 units of contaminant at $t=0$, at which time air in the subway station was static (air velocity was uniformly 0 at all location). At this point, a train model of $1 \mathrm{~m}$ by $1 \mathrm{~m}$ with the speed of $1 \mathrm{~m} / \mathrm{s}$ moved from left to right. After $8 \mathrm{~s}$ the train completely passed through, another train with the same speed passed through from right to left. The whole process took totally 16 seconds. There are two sensors installed in the space, which are attached on the opposite wall to detect the concentration of the contamination in the station continuously. Figure 2 gives the actual position of both sensors and source.

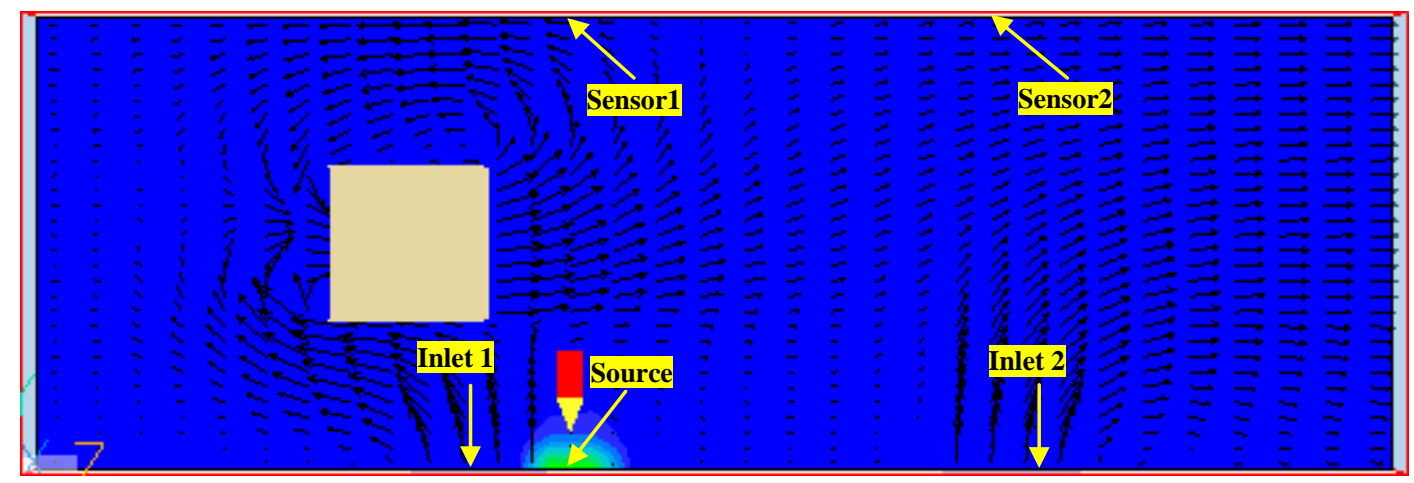


Figure 2. Configuration, contaminant source, and sensor locations of the subway station CFD was first employed to run a regular unsteady simulation in order to obtain the airflow and contaminant concentration data under dynamic airflow in the subway station. The airflow velocity fields of all grid cells in each time step is stored. Contaminant concentration value that represent sensor readings in physical experiment at the position of sensors, which can be recorded for the future inverse modeling. . In the forward CFD simulation, the structured grid size of $0.27 \times 0.1 \mathrm{~m}$ was used for this subway station. The unsteady CFD simulation was specified to have the 8 time steps and each time step is $2 s$. Standard $k$ - $\varepsilon$ model[40] was used for the turbulent modeling.

The contaminant source is released under the dynamic air flow from $t=0 s$, and the concentration of the contaminant is monitored and recorded by sensors. This is a typical contaminant monitoring and measurement configuration in reality, and it is modeled by regular CFD method in this study. As shown in Figure 3, the vector represents the velocity, and the contour represents the contaminant concentration field, the white arrow within the block represents the direction of the train. Obviously air velocity in the domain changes from time to time, which is typical for subway station.

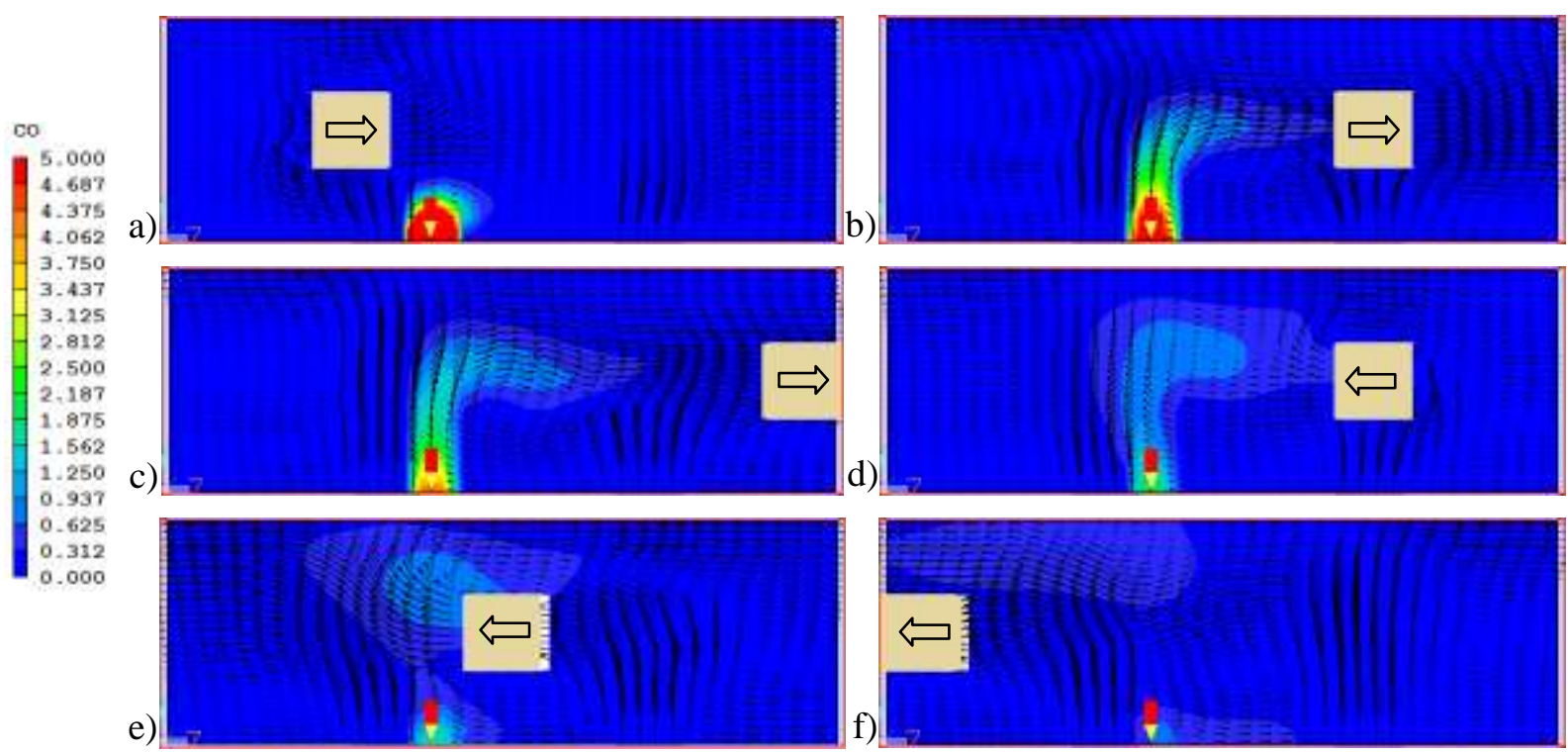

Figure 3. Predicted airflow field and the concentration of the contaminant by forward CFD simulation at a) $t=2 s, b) t=6 s, c) t=8 s, d$ ) $t=10 s, e) t=12 s, f) t=16 s$. 


\section{The original adjoint probability method for identifying an instantaneous airborne contaminant location}

Assume that the change of the air flow caused by the train passing through is not considered in a research, and only the air conditioning system supply air is considered, which is, the airflow within the station platform is stable. Such a stable airflow field data is utilized for inverse modeling in the original adjoint probability method. The historical concentration readings presented in Figure 3 are used as input. Figure 4 presents the predicted CALP distribution under such steady airflow by employing the original adjoint probability method by using different sensor readings as input.

As illustrated by Figure 4. a), the CALP distribution in the domain is rather dispersive when two one-time readings of Sensor 1 were utilized. The small dark triangle represents the actual contaminant source location in this case. The location which has the highest probability value was close to the Sensor 1 location. Obviously, the predicted CALP distribution under such steady airflow by employing the original the adjoint probability method is wrong. Figure 4 . b) using three one-time readings as input was not able to find the actual contaminant source location either. As shown in Figure 4. c) and 4. d), which uses readings from Sensor 2, the simulation results in the domain is rather dispersive and can't predicted the actual source location, verifying that the original adjoint probability method is unfit for source location identification when environment airflow goes dynamic.

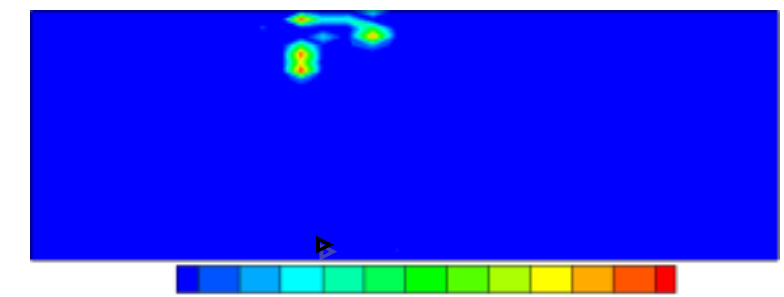

a)

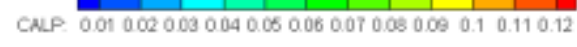

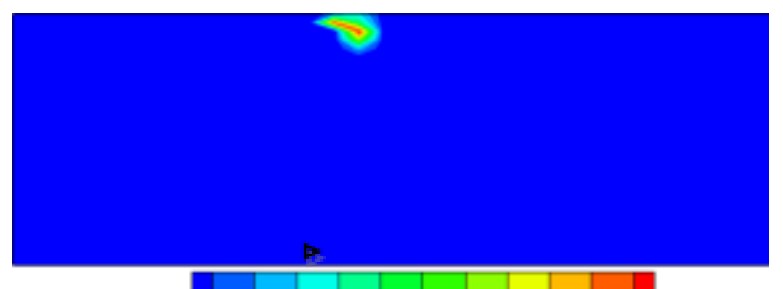

b)

CALP. 0.020 .040 .060 .080 .10 .120 .140 .160 .180 .20 .22 

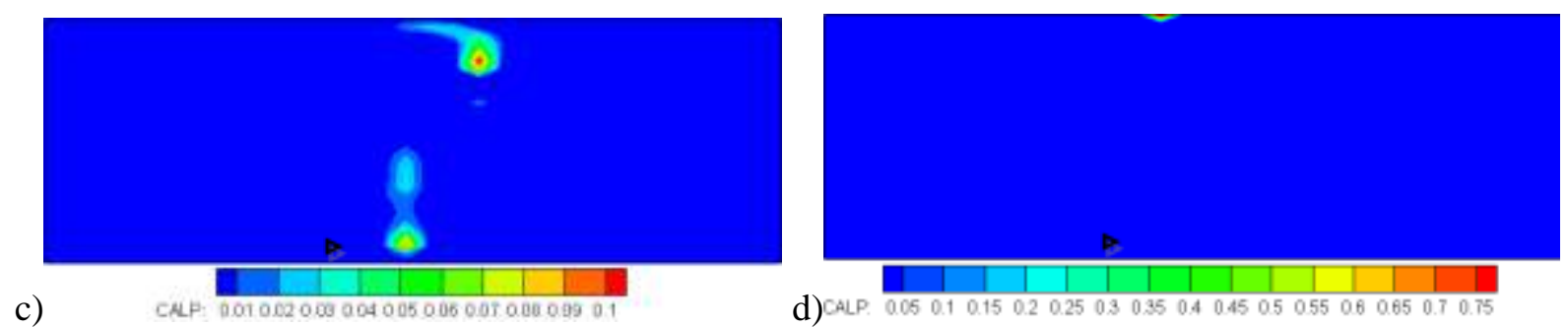

Figure 4. Predicted position of contaminant source probabilities by the original adjoint probability method for the subway station case with a) two one-time readings of Sensor 1, b) three one-time reading of Sensor 1, c) three one-time readings of Sensor 2, d) both sensors with historical concentration readings

\section{The new mathematical model for identifying an instantaneous airborne contaminant}

\section{location under dynamic airflow}

The newly developed mathematical model was used for identifying an instantaneous airborne contaminant location under dynamic airflow for the same scenario. Airflow field data in each time step has been utilized in such new algorithm. The contaminant concentration readings of Sensor 1 and Sensor 2 can be obtained by solving Eq. (7). This forward simulation is a counterpart of physical experiment. In practice, sensors monitoring certain airborne contamination will provide such concentration data. As shown in Figure.1, to identify the airborne contaminant source location, SALP should be obtained by solving Eq. (8). Figure 5 presents the predicted CALP distribution under such dynamic airflow by solving Eq. (10) and (11) introduced in this paper by using different sensor readings as input. As illustrated by Figure 5. a), the CALP distribution in the domain is rather dispersive when two one-time readings of Sensor 1 was utilized. The small dark triangle represents the actual contaminant source location in this case. Though the highest probability area which indicates the most probable source location, was close to the real position of contaminant source, visible probability value also appeared in other location. Figure 5. b) using three one-time readings as input is more concentrated than result of Figure 5.a), and the source position identification is very closer to the actual source releasing position. 
As observed from forward simulation, Sensor 2 has relatively small and late concentration reading values compare to Sensor 1 during the contaminant transportation process. So the performance of source identification is worse than Sensor 1. As shown in Figure 5. c), which uses 3 one-time readings from Sensor 2, the highest source location probability (about 15\%) was found near actual source position, which matches the testing scenario. Figure 5. d) shows the simulation results by solving Eq. (10) and Eq. (11) with three readings by both Sensor 1 and Sensor 2, which are very similar to those in Figure 5. b). Under the newly developed mathematical model, the results of source location identification are very closer to the actual source releasing location, verifying the applicability of the algorithm in this case.

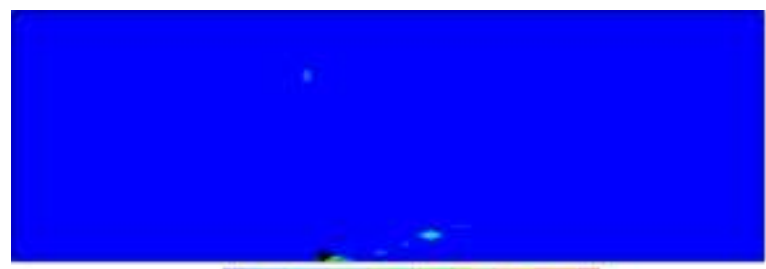

a) CALP. 000000010.120140 .160380 .222

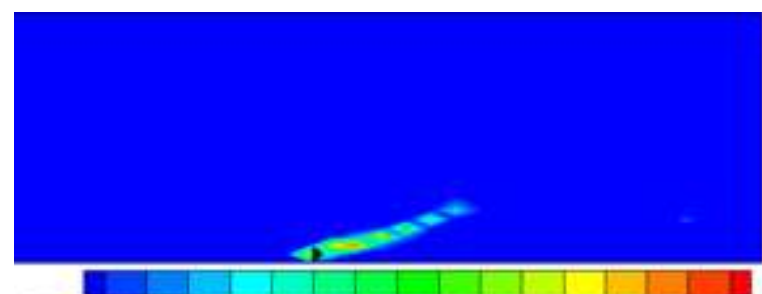

c)

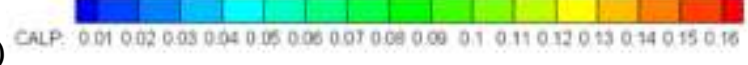

d)

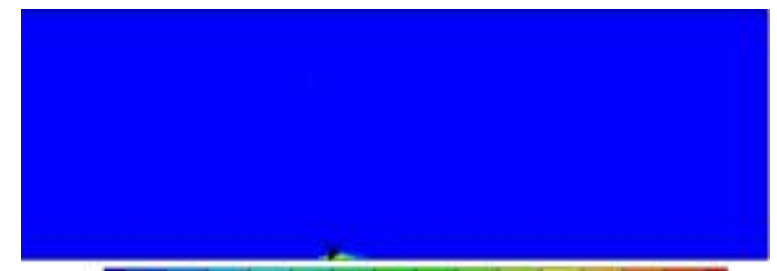

b)

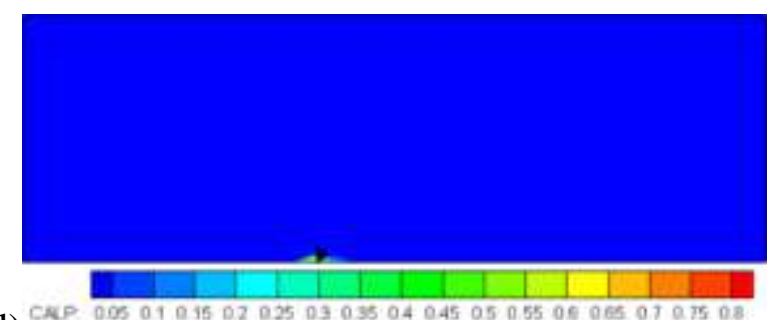

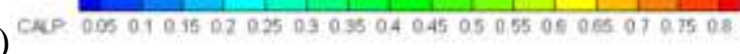

Figure 5. Predicted position of contaminant source probabilities by the joint probability method for the subway station case with a) two one-time readings of Sensor 1, b) three onetime reading of Sensor 1, c) three one-time readings of Sensor 2, d) both sensors with historical concentration readings

\subsection{Air contamination in an aircraft cabin}

\section{Numerical method}

At present, there are mainly three types of air supply system in an aircraft cabin: inlets at the top of the cabin, inlets at the side walls of the cabin, and combination of the previous two.

Side wall supply system has inlets located below the luggage racks with very low supply air 
velocity such that the passengers can obtain a comparatively comfortable cabin environment. Therefore, this air supply system is commonly used in the cabin. Li and Liu [41] found that this mode of supplying air at side walls and exhausting in both sides near the floor will result in two-jet collision regions with unsteady characteristic in cabin through PIV test. This is very typical in the sense that a fixed boundary condition results in dynamically changing airflow field in a built environment. As shown in Figure 6, the cabin mock-up was designed as a replica of a 7-row section of the main body of a Boeing 737-200, which was occupied by 42 thermal manikins (42 seats). The heat load of each manikin was $75 \mathrm{~W}$.

The dimensions of the cabin at floor level were $5.80 m(\mathrm{Y}) \times 3.25 \mathrm{~m}(\mathrm{X})$, and at luggage carrier level, they were $5.80 \mathrm{~m}(\mathrm{Y}) \times 1.26 \mathrm{~m}(\mathrm{X})$. The heights were $2.15 \mathrm{~m}(\mathrm{Z})$ and $1.60 \mathrm{~m}$ (Z)from the floor to the ceiling and to the bottom of the luggage compartments, respectively. Air diffusers were placed along each side at the luggage compartment level along each side. The dimensions of air diffusers were $5.80 m(\mathrm{Y}) \times 0.023 m(\mathrm{Z})$. Air was supplied from the lateral diffusers into the cabin with relatively symmetrical boundary conditions with a velocity of approximately $1.8 \mathrm{~m} / \mathrm{s}$. Air outlets were located at both sides of wall near the floor. In this study, Computational Fluid Dynamics (CFD) tool was used to explore the unsteady characteristics in cabin. In CFD simulation, RANS models (Reynolds averaged Navierstokes) and LES (Large eddy simulation) model are widely selected for investigate the unsteady flow distribution in indoor environment study. The computing time of LES is much longer than RANS models, although LES performs better in simulation of the unsteady flow characteristics. Unsteady RANS models is another option for dynamic airflow field simulation as shown in previous subway station case, RANS models were found to have the ability to describe the characteristics of the flow collision zones[42]. Both $2 \mathrm{~d}$ and $3 \mathrm{~d}$ RNG k-e models are proved to be able to describe the flow collision characteristics of an aircraft mockup[43]. This paper, RANS models was used to simulate two-jet collision regions with strong 
uncertainty and unsteady characteristics in cabin. It is a typical dynamic air flow environment for identifying an instantaneous airborne contaminant location.

CFD was first used to run an unsteady simulation to obtain the airflow and contaminant concentration data under dynamic airflow. The velocity field data can be obtained in each grid cell for all time steps. Contaminant concentration value at the position of sensor can be recorded in each time step for the inverse modeling purpose. In the forward CFD simulation, the structured grid size of $0.08 \mathrm{~m}(\mathrm{X}) \times 0.18 \mathrm{~m}(\mathrm{Y}) \times 0.05 \mathrm{~m}(\mathrm{Z})$ was used for this cabin. The unsteady CFD simulation was specified to have the 20 time steps and each time step was $2 s$.

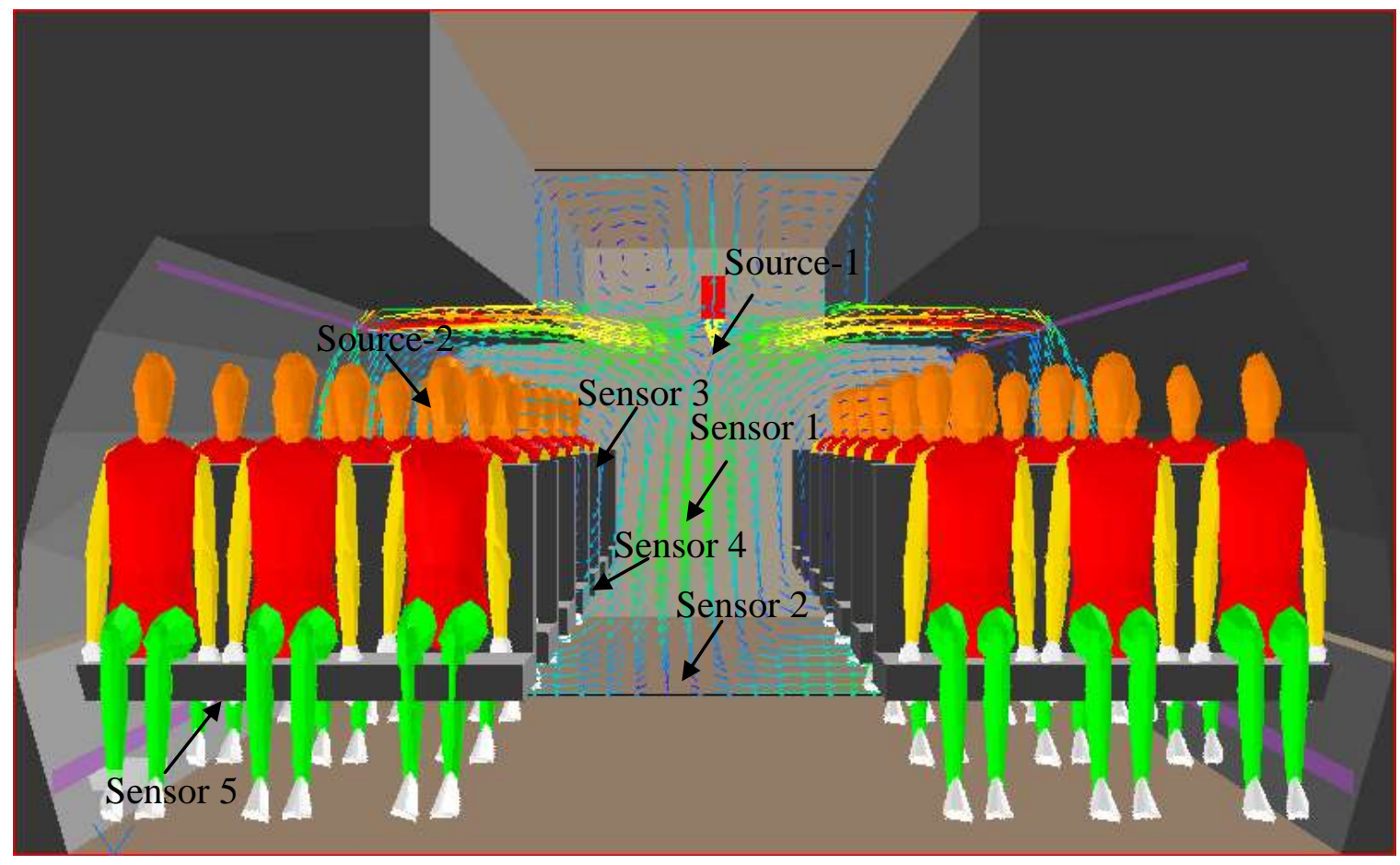

Figure 6. Configuration of the airplane cabin model

There is a point contaminant source at the position of red arrow released 100 units of contaminant at $t=0$, at which time air in the cabin was static (air velocity is uniformly 0 at all location). Five sensors were installed in the cabin within the same intersection right in front of the passenger of the third row. This is because airflow velocity in transverse direction is so small as to make it close to a two-dimensional flow[44]. Figure 6 shows the locations of the contaminant source and the sensors, which can detect the concentration of the contaminant 
continuously. Two different tests as in Table 1 were conducted. The detailed location of sources and sensors, using bottom left of the figure as origin of the coordinate, is listed in Table 2.

Table 1 Indoor contamination test scenarios

\begin{tabular}{|c|c|c|c|}
\hline Sensing scenario & Source index & Sensor index & Concentration reading type \\
\hline 1 & 1 & 1,2 & Historical reading \\
\hline 2 & 2 & $3,4,5$ & Historical reading \\
\hline
\end{tabular}

Table 2 The position of sensors and sources

\begin{tabular}{|l|l|l|l|}
\hline Index & $\mathrm{X}(\mathrm{m})$ & $\mathrm{Y}(\mathrm{m})$ & $\mathrm{Z}(\mathrm{m})$ \\
\hline Source 1 & 0.75 & 2.78 & 1.2 \\
\hline Source 2 & 1.80 & 2.78 & 1.42 \\
\hline Sensor 1 & 1.71 & 2.78 & 0.78 \\
\hline Sensor 2 & 1.71 & 2.78 & 0.05 \\
\hline Sensor 3 & 1.50 & 2.78 & 1.05 \\
\hline Sensor 4 & 1.50 & 2.78 & 0.45 \\
\hline Sensor 5 & 0.30 & 2.78 & 0.15 \\
\hline
\end{tabular}

Verification of necessity of using the new method for indoor contaminant source identification under dynamic airflow.

Since this aircraft cabin case is special that a fixed boundary condition cause dynamic indoor airflow in built environment, researchers or engineers may take the convenience to model such a case using steady CFD method hence obtain a steady-state airflow field and apply it to inverse modeling by original adjoint probability method. Whether this will work properly has significant practical implication. Therefore, this was tested by applying the two different methods. First of all, Scenario 1 as listed in Table 1 was used to compare the results of two methods to identify an instantaneous airborne contaminant location. As a comparison scenario, it did not consider whether the location of the source and the sensor is reasonable in practical application. 
As illustrated in Figure 7. a), predicted position of contaminant source probabilities by the original adjoint probability method for the airplane cabin case with Sensor 1 using a steadystate airflow field, the small dark triangle represents the actual position of contaminant source in this scenario. The CALP distribution in the domain is completely deviated from the right location comparing to the actual contaminant source location in this scenario. Figure $7 . \mathrm{b}$ ), Predicted source location probabilities with Sensor 1 under dynamic airflow, the CALP distribution in the domain was much closer to the real location. The highest probability area was close to the actual location. Obviously, the predicted CALP distribution under dynamic airflow is more appropriate than under steady airflow for the Sensor 1. Figure 7. c) using historical concentration readings of Sensor 2 with steady airflow as input couldn't find the actual contaminant source location in this case. The CALP distribution in the domain was similar to Figure 7.a). As shown in Figure 7. d), which uses readings from Sensor 2 under dynamic airflow, the simulation results in the domain was approaching the actual source location. The above investigation and analysis, verified the invalidity of the original adjoint probability method and necessity of the new method for indoor contaminant source location identification under dynamic airflow in this case.
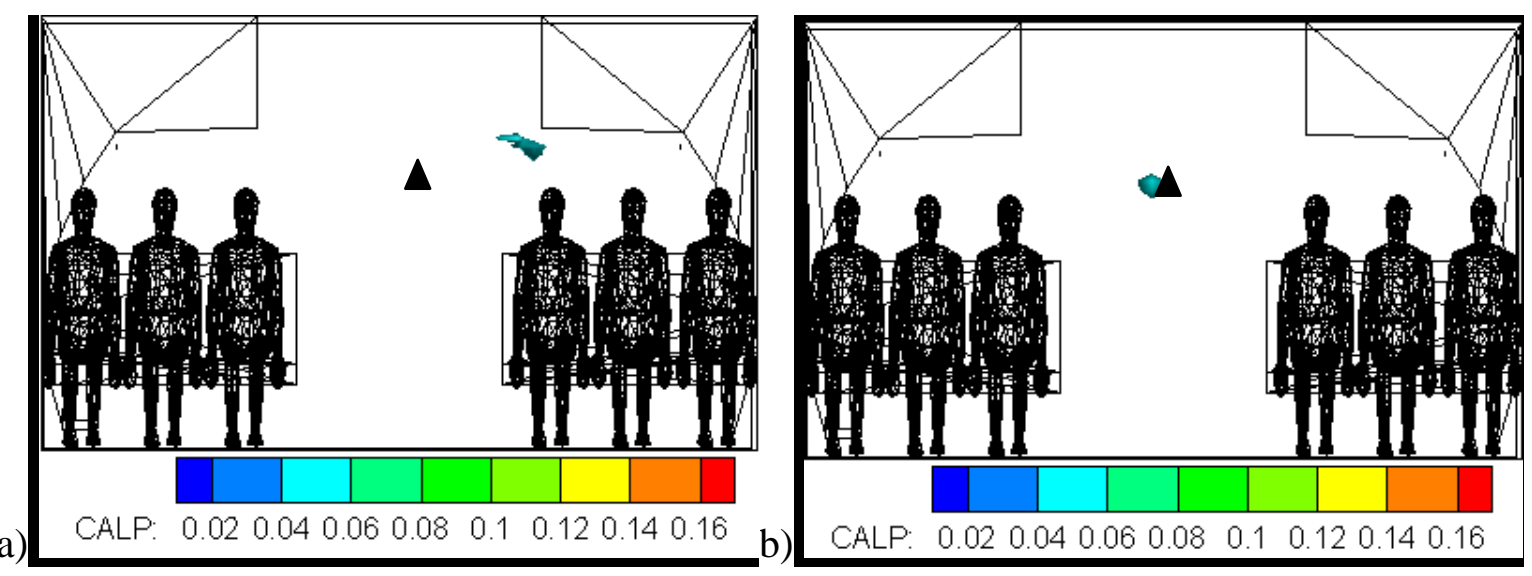

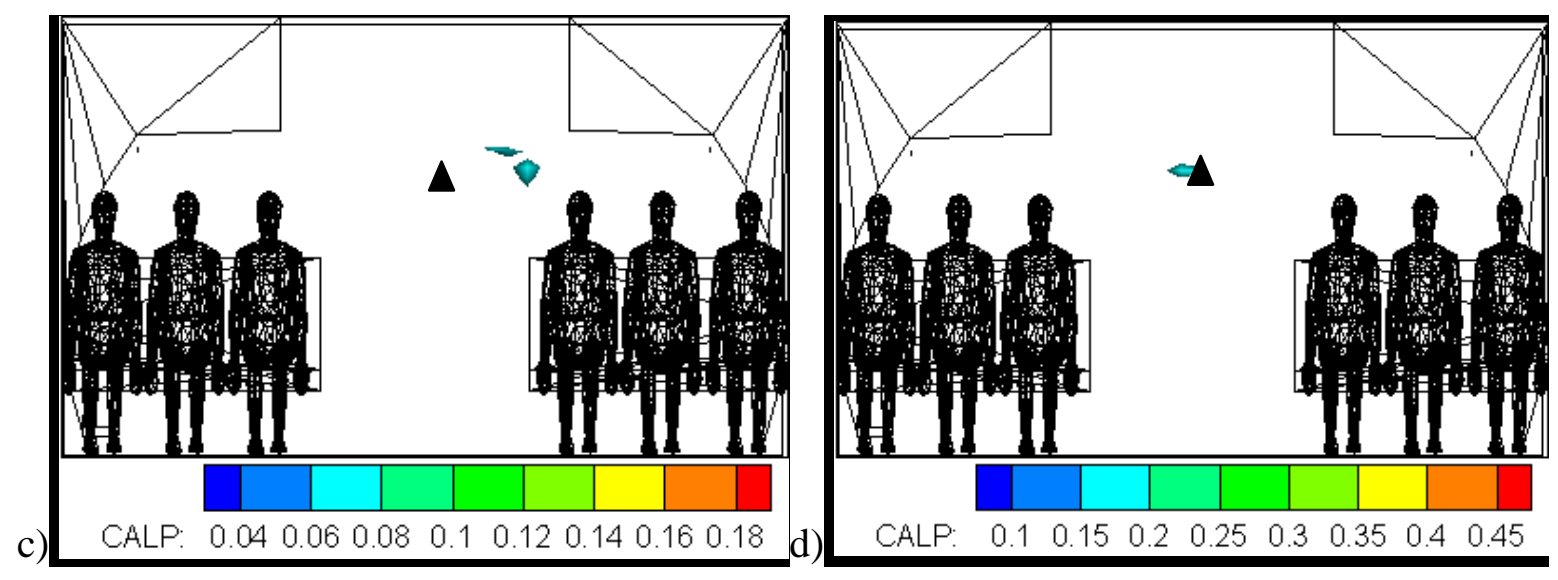

Figure 7. Predicted position of contaminant source probabilities by the adjoint probability method for the cabin case with a) historical concentration readings of Sensor 1 under steady airflow (original method), b) historical concentration readings of Sensor 1 under dynamic airflow (new method), c) historical concentration readings of Sensor 2 under steady airflow (original method), d) historical concentration readings of Sensor 2 under dynamic airflow (new method)

Performance of the new mathematical model for identifying an instantaneous airborne contaminant location under dynamic airflow

With the above analysis, necessity of the new method for indoor contaminant source identification under dynamic airflow is verified. However, different source and sensor location configuration can affect the accuracy of inverse modeling result significantly. Scenario 2 of Table 1 for identifying an instantaneous airborne contaminant location under dynamic airflow is further investigated. The second passenger on the fourth row was assumed to be contaminant source, releasing infectious airborne virus from coughing. Three virus sensors were installed on the aisle side of the third passenger seats and the left side wall of the slot outlet. All of these are within the same intersection. 

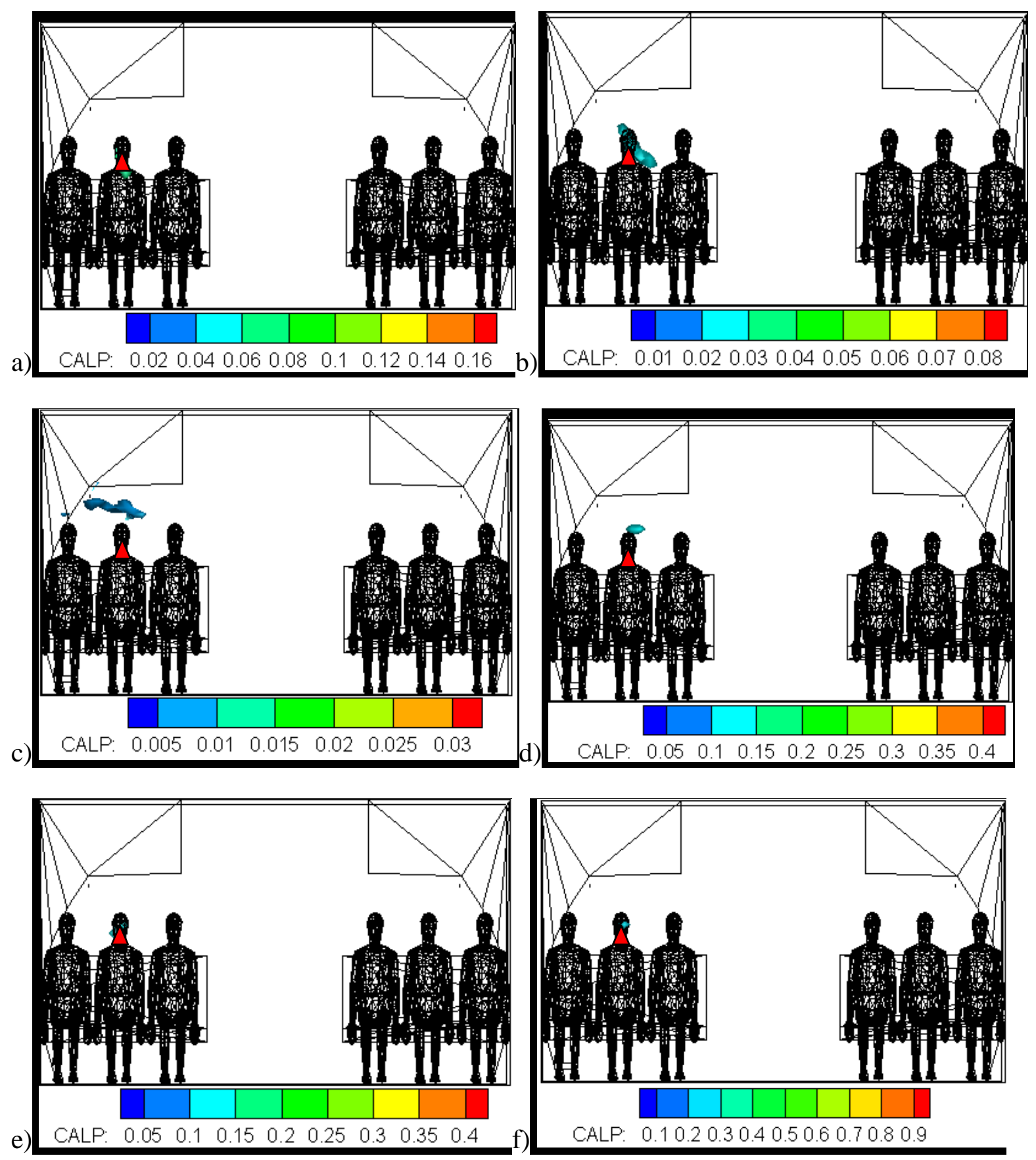

Figure 8. Predicted contaminant source position probabilities by the newly developed adjoint probability method for the cabin case with a) historical concentration readings of Sensor $3, b$ ) historical concentration readings of Sensor 4, c) historical concentration readings of Sensor 5, d) multiple historical concentration readings of Sensor 4 and Sensor 5, e) multiple historical concentration readings of Sensor 3 and Sensor 5, f) multiple historical concentration readings of three sensors

Following the same procedure as illustrated by Figure 1, source location probability is computed. Figure 8, presents the predicted CALP distribution under such dynamic airflow by solving Eq. (10) and Eq. (11). As illustrated by Figure 8. a), the accuracy of predicted results 
were improved when historical concentration readings of Sensor 3 as input, the small red triangle represents the actual contaminant source location in this scenario. The highest probability area was predicted close to the real source position. Figure 8 . b) using historical concentration readings of Sensor 4 as input is similar to Figure 8. a), which the identified source position is close to the actual position. As observed from forward simulation, Sensor 5 has relatively small and late concentration reading values compared to the Sensor 3 and Sensor 4 during the contaminant transportation process. So the performance of source identification is worse than other sensors. As shown in Figure 8. c), which uses historical concentration readings from Sensor 5, the CALP distribution in the domain is rather dispersive. Though the highest probability area was close to the actual source location, visible probability value showed up in other location. Figure 8. d) shows the simulation results with multiple historical concentration readings of Sensor 4 and Sensor 5, which are more concentrated than the previous results. Sensor 5, although only detects relatively small value of contaminant concentration, can still help to improve the accuracy of the contaminant source location identification, as can be observed from the prediction results in Figure 8. d). Figure 8. e) is similar to Figure 8. d). Figure 8. f) shows the identification results with multiple historical concentration readings of three sensors, the identified source position is very close to the actual source position.

On the other hand, by comparing Figure 8. a), b), c), we can find that figure 8. a) has the best prediction of the source location, figure $8 . \mathrm{b}$ ) take the second place, and figure 8 . c) has the worst performance. This is due to the different positions of the three sensors. Figure 6 shows that Sensor 3 is located above the seat, in the downstream of the source location, and the distance from the pollution source is relatively small, so the response time and the readings of sensor are relatively short and large. This is possibly the reason that Sensor 3 has the best prediction of the source location. The position of Sensor 4 under the seat, in the downstream 
of air flow far from source location, and part of the flow is blocked by the seat. As a result, Sensor 4 has a longer response time and smaller contaminant concentration reading values compared to the Sensor 3. So the performance of source identification is worse than Sensor 3. Sensor 5 is located in the left side of the air outlet far from source location in the downstream of the air flow, and the response time is the longest among all three situations. So the effective information obtained by the Sensor 5 is the least, which only detects relatively small value of contaminant concentration, which makes the source location prediction of Sensor 5 is the least accurate among these three sensors. Through above analysis, it can be concluded that the position of sensors on the source location identification is very crucial. An optimal sensor network should be designed to provide efficient and effective information for contaminant source identification [17] [45] [46] [47]. In the real case scenario, general airflow pattern and anticipated location of potential contaminant source prior to occurrence of such incidences usually helps the configuration of proper sensor placement.

\section{Experimental Validation}

In order to validate application of the adjoint method using physically measured contaminant concentration data, experiment using a full-scale aircraft cabin mock-up, which is the prototype of previously mentioned simulation based on a Boeing 737-200 cabin was conducted. Detail description of this mock-up was introduced in simulation section. All boundary conditions used in CFD simulation were measured from this facility. The picture taking from outside of the mock-up is in Figure 9. 


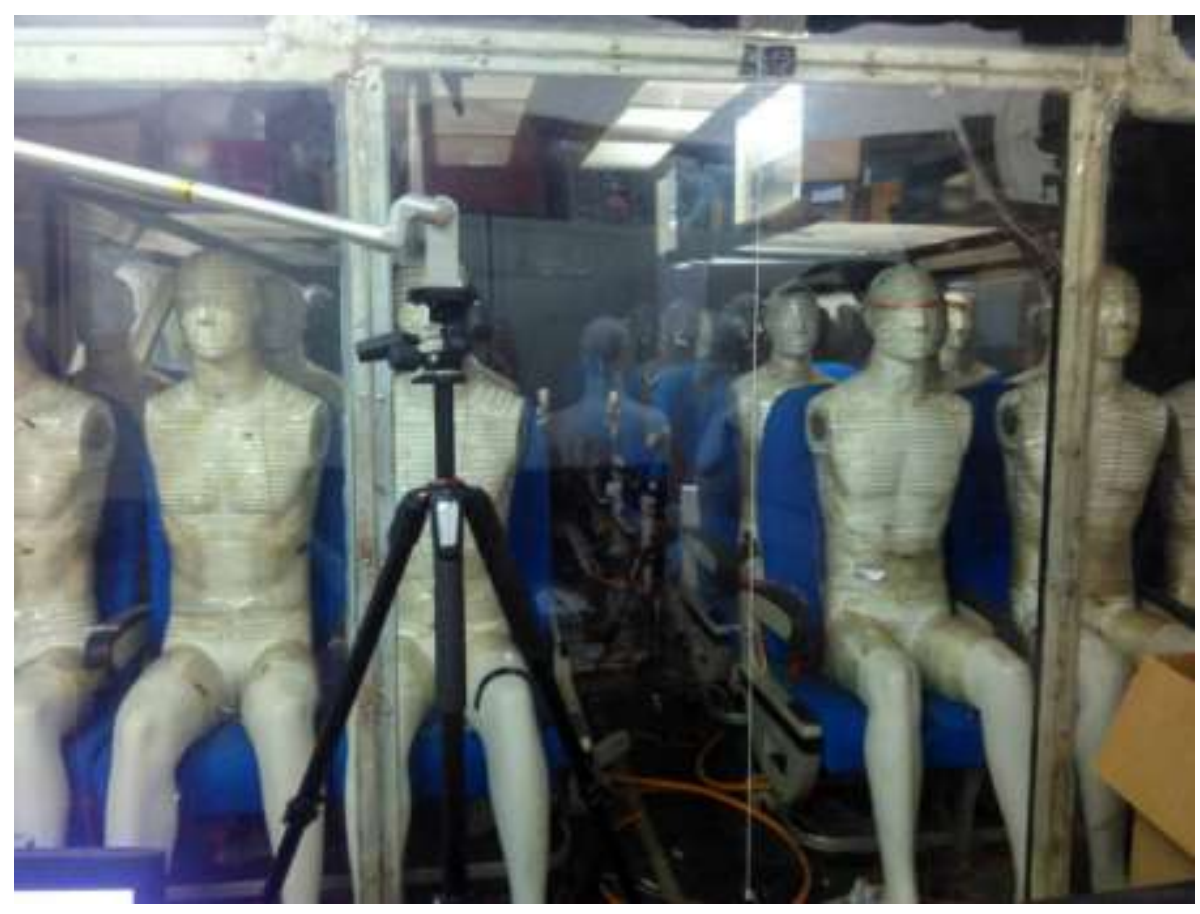

Figure 9. The outside view of full scale aircraft cabin mock-up

In this experiment, carbon dioxide $\left(\mathrm{CO}_{2}\right)$ was selected as the tracer gas to simulate airborne contaminants breathed out from sick passengers. A specific passenger released the gaseous contaminant at the breathing zone at the rate of $20 \mathrm{~L} / \mathrm{min}$, for 10 seconds (one time-step as used in CFD simulation to represent instantaneous release). The location of the contaminant source and the sensors were shown in scenario 2 in Table 1 . Infrared $\mathrm{CO}_{2}$ sensors (Telaire ${ }^{\circledR} \mathrm{T}$ 7001) were used to monitor the concentration. The accuracy of the sensor is $\pm 50 \mathrm{ppm}$. The background concentration of $\mathrm{CO}_{2}$ in ambient environment was $363 \mathrm{ppm}$.

The flow pattern attained from simulation should be similar to the actual situation to make sure that the CFD model can successfully predict the source location. However, the process to obtain the actual flow field and perform a comparison between the simulated scenario and actual flow field for the whole cabin is really time-consuming and costly. An alternative option by performing a contaminant concentration comparison can make sure that the air flow filed of simulation is consistent with the experiment. Since the airflow inside the cabin has periodic oscillation characteristics, different contaminant releasing time may cause different 
concentration distribution. Therefore, the $\mathrm{CO}_{2}$ concentration variation may have a certain phase difference between simulation and measurement on the same sensor. In the experiment, $\mathrm{CO}_{2}$ was released to the cabin at a random time. The $\mathrm{CO}_{2}$ concentration of Sensor 3 and Sensor 5 between simulation and measurement were picked for such comparison as plotted in figure 10. The concentrations from the CFD model reached an acceptable agreement with those from the experiment, as revealed by Figure 10. These $\mathrm{CO}_{2}$ concentrations were then used as inputs of the inverse modeling program in order to pinpoint the source location.

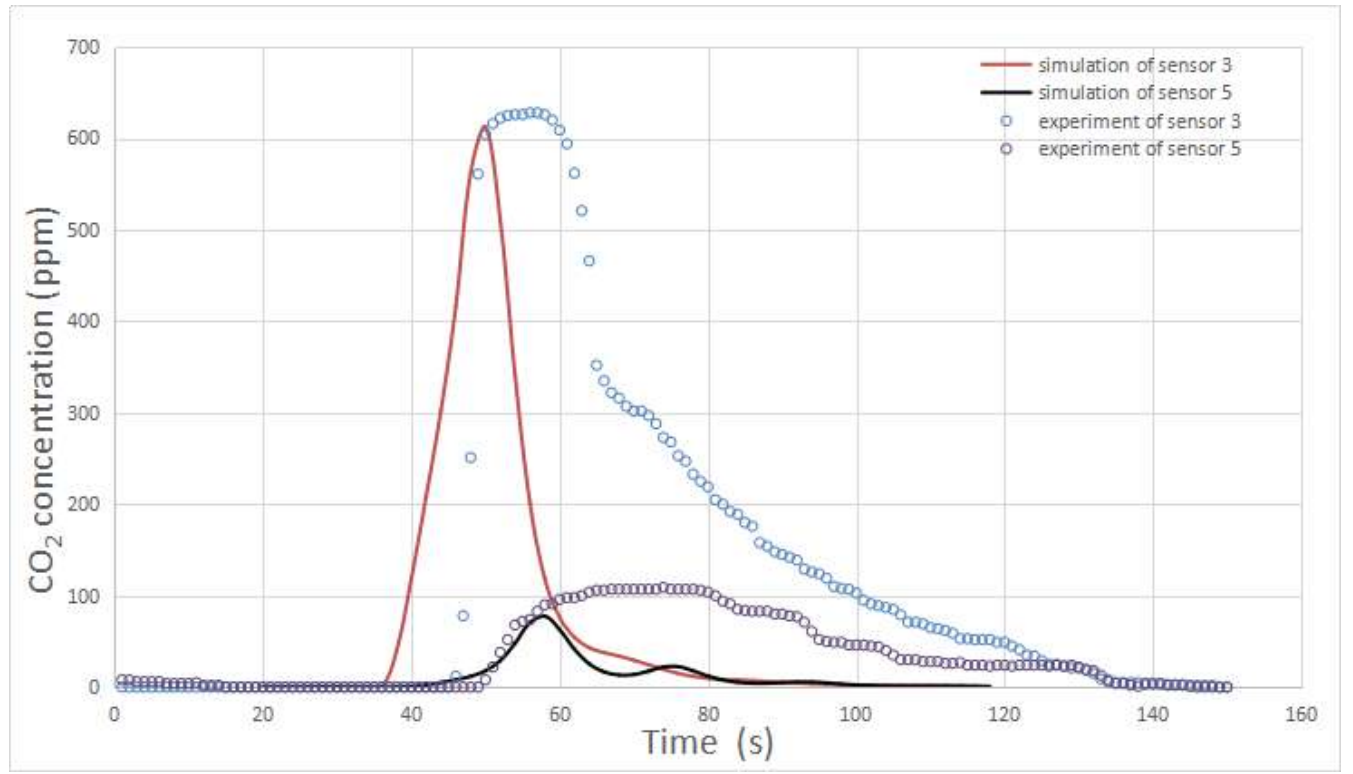

Figure 10. Comparison of the concentration (background concentration deducted) of Sensor 3 and Sensor 5 between the CFD model and the experiment

The apparatus used to monitor the $\mathrm{CO}_{2}$ concentration in the experiment requires a certain response time (less than 20s), plus that $\mathrm{CO}_{2}$ releasing time may have certain phase difference with simulation, which causes certain phase difference between two data sets. However, the proposed adjoint method which used in this paper is to solve the adjoint probability of contaminants concentration distribution at different moments, so the phase difference has little influence on the accuracy of the positioning results. Details about the source positioning results are presented in Figure 11. 

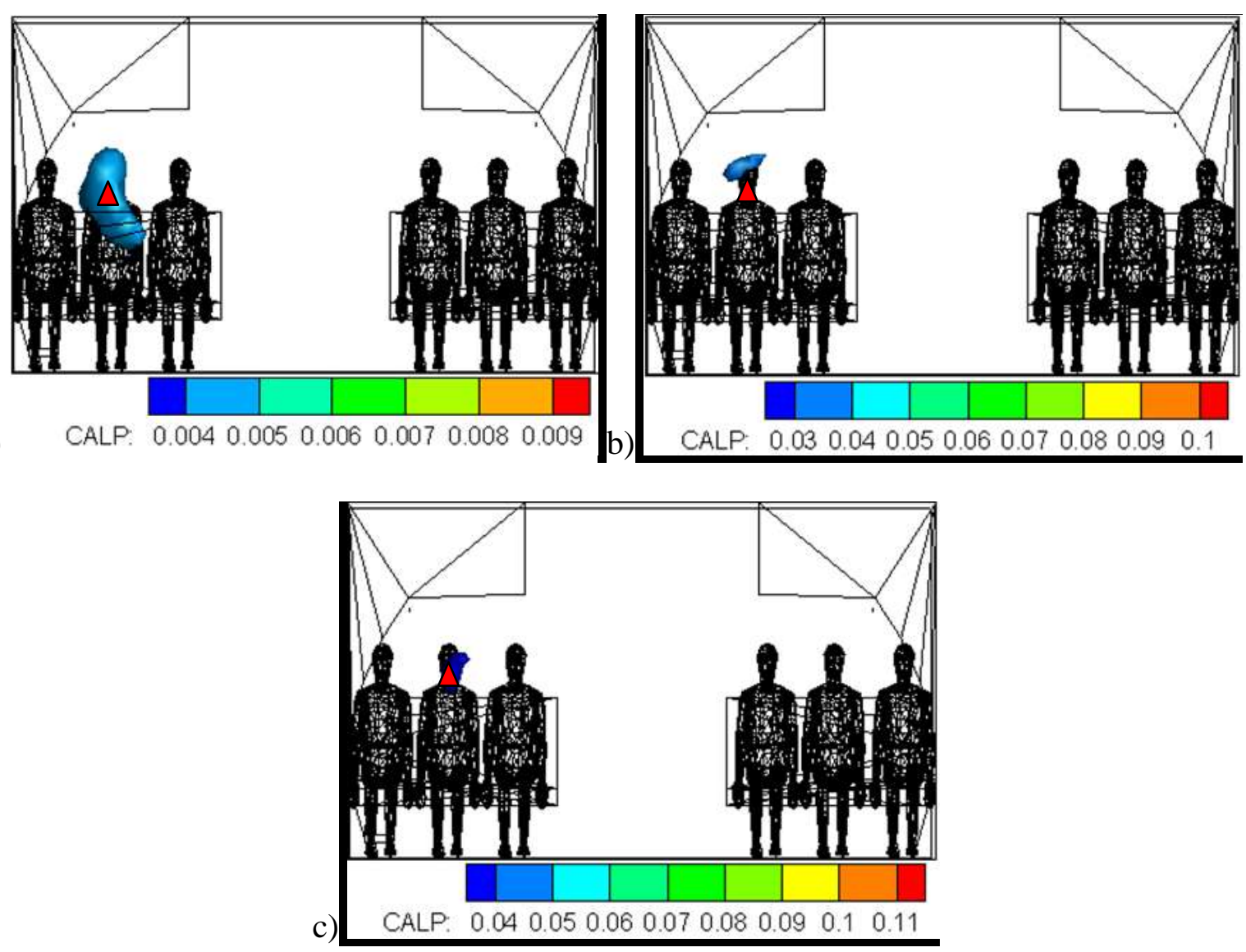

Figure 11. Predicted contaminant source position probabilities by the newly developed adjoint probability method for the cabin case with a) historical concentration readings of Sensor 3; b) multiple historical concentration readings of Sensor 3 and Sensor 5; c) multiple historical concentration readings of all three sensors

As illustrated by Figure 11. a), the results of contaminant source position identification were when historical concentration readings of Sensor 3 from the experiment was used as input, and the small red triangle represents the actual contaminant source location in this scenario. Although the identified possible source location is dispersed in a large area, the possible location is near the actual source of manikin. Figure 11. b) showed the simulation results with multiple historical concentration readings of Sensor 3 and Sensor 5, which were more concentrated than the Figure 11. a). Sensor 5, although only detected relatively small value of contaminant concentration, can still contribute to improve the accuracy of contaminant source location identification. Figure 11. c) showed the simulation results with multiple historical concentration readings of three sensors, the identified source position was very close to the actual position. 


\section{DISCUSSION}

Generally a satisfactory CALP result was obtained, for both subway station and aircraft cabin case, with both simulated concentration and measured concentration as input, to verify the new adjoint method in identification of airborne contaminant source location under dynamically changing airflow field, even though different sensor locations and their combinations exhibited different performance and confidence in identifying the actual source location. The absolute value of CALP is an indicator of confidence on the identified source location. Although all case studies identified the correct contaminant source location with the peak relative CALP value by carefully tweaking sensor input, it is affirmative that sensor location and which sensor readings to use is critical for identifying the source location with this method.

In practice, obtaining the transient flows by experiment is quite difficult and also very expensive. CFD method with unsteady RANS simulation provides an alternative to obtain real-time airflow field data provided that enough computational resource is available. This can be achieved by monitoring real-time boundary condition of a specific case. However, the difference between simulation and real physical condition of velocity is a potential cause of error. In this proposed method, an alternative option by performing a contaminant concentration comparison is to make sure that airflow field of simulation is consistent with the experiment. However, according to [48], air velocity accuracy is not strictly required for source location identification. As long as general airflow pattern especially vortex shape of CFD simulation is similar to the actual flow pattern, it is enough for an accurate source location prediction.

The proposed method in finding the location of an instantaneous source assumes a known releasing time. However, in a real IAQ event, prior information about the source may not be enough and there are many different cases such as continuously released source, multiple 
source locations and so on. Sometimes both location and release time need to be found. Sometimes most sources release contaminants more or less continuously, such as chemical being released from building materials, furnishings. This impose a great challenge for contaminant source identification. This is also our main research direction in the future.

\section{CONCLUSIONS}

This paper introduced the mathematical model of the indoor instantaneous contaminants source location identification under dynamic air flow scenario and developed a CFD-based inverse source tracking algorithm. With airflow field data of each time step and certain concentration readings obtained by contaminant sensor(s), source location can be predicted. The algorithm applicable to unsteady flow cases were demonstrated and verified by two example cases: a two-dimensional subway station case and a three-dimensional aircraft cabin case, with both simulated and measured concentration data. It is found that previously developed adjoint method for steady-state airflow field failed to identify indoor airborne contaminant location under such dynamic airflow field. The new algorithm considering the airflow field variation is necessary for airborne contaminant source location identification. Case studies indicate that sensor location and input concentration selection is critical for correctly pinpointing the source location. The numerical and experimental case studies verified the feasibility, effectiveness and accuracy of the method for predicting the position of indoor contaminant source under dynamic airflow scenario. The practical implication of this study is: CFD tool using unsteady RANS simulation can be used to obtain real time airflow field information by monitoring time-dependent boundary condition, with proper sensor installed to monitor contaminant concentration readings, the new developed algorithm can be used to pinpoint such air contaminant source location.

\section{Acknowledgements}


Authors of this paper would like to express their gratitude to Professor Junjie Liu for providing aircraft cabin mock-up for experimental validation of this research.

Funding: This work is sponsored by National Science Foundation of China (51508326) and supported by Shanghai Pujiang Program (15PJ1406300).

\section{REFERENCES}

[1]Sarvesh Kumar Singh, Maithili Sharan, Jean-Pierre Issartel, Inverse Modelling for Identification of Multiple-Point Releases from Atmospheric Concentration Measurements, Bound-Layer Meteorol. 146(2013) 277-295.

[2]Jean Pierre Issartel, Maithili Sharan, Sarvesh Kumar Singh, Identification of a Point of Release by Use of Optimally Weighted Least Squares, Pure Appl. Geophys. 169(2012)467-482.

[3]Animesh Khemka, Charles A. Bouman, Mark R. Bell, Inverse problems in atmospheric dispersion with randomly scattered sensors, Digit. Signal Process. 16 (2006) 638-651.

[4]Volkan Akcelik, George Biros, Andrei Draganescu, Omar Ghattas, Judith Hill, Bart van Bolemen Waanders, Inversion of Airborne Contaminants in a Regional Model, Comput. Sci. - ICCS. (2006) 481-488.

[5]P. Kathirgamanathan, R. McKibbin, R.I. McLachlan, Source Release-Rate Estimation of Atmospheric Pollution from a Non-Steady Point Source at a Known Location, Environ. Model. and Assess. 9( 2004)33-42.

[6]P. Kathirgamanathan, R. McKibbin, R.I. McLachlan, Source release rate estimation of atmospheric pollution from a non-steady point source-Part 2: Source at an unknown location,Res. Lett. Inf. Math. Sci. Vol.5( 2003) 85-118.

[7]Shungo Kato, Pakpong Pochanart, Jun Hirokawa, The influence of Siberian forest fires on carbon monoxide concentrations at Happo, Japan, Atmos. Environ. 36 (2002) 385-390.

[8]Tian-hu Zhang, Xue-yi You, A simulation-based inverse design of preset aircraft cabin environment, Build. and Environ. 82(2014) 20-26.

[9]Liu X. and Zhai Z. Inverse modeling methods for indoor airborne pollutant tracking: literature review and fundamentals. Indoor Air. 17;6 (2007) 419-438.

[10]Alapati S. and Kabala Z. J. Recovering the release history of a groundwater contaminant using a non-linear least-squares method. Hydrol. Process. 14;6 ( 2000) 1003-1016.

[11]Mahar P. S. and Datta B. Identification of pollution sources in transient groundwater systems. Water Resour. Manag. 14;3 (2000) 209-227.

[12]Mahar P. S. and Datta B. Optimal identification of ground-water pollution sources and parameter estimation. J. Of Water Resour. Planing and Manag.-ASCE 127;1 (2001) 20-29.

[13]Skaggs T. H. and Kabala Z. J. Recovering the history of a groundwater contaminant plume: method of quasi-reversibility. Water Resour. Res. 31;11 (1995) 2669-2673.

[14]Cornacchiulo D., Bagtzoglou A.C. and Atmadja J. Hydrologic inverse on using marchingjury backward beam equation and quasi reversibility methods. 2002; In: Proceedings of 
the 15th ASCE Engineering Mechanics Conference. New York City, NY, Columbia University.

[15]Liu X. and Zhai Z. Protecting a whole building from critical indoor contamination with optimal sensor network design and source identification methods. Build. Environ. 44;11 (2009) 2276-2283.

[16]Michael D. Sohn, Pamela Reynolds, Navtej Singh, Ashok J. Gadgil, Rapidly Locating and Characterizing Pollutant Releases in Buildings, J. of the Air \& Waste Manag. Assoc. 52 (2002) 1422-1432.

[17]Sreedharan P, Sohn M, Gadgil A, Nazaroff W, Systems approach to evaluating sensor characteristics for real-time monitoring of high-risk indoor contaminant releases, Atmos. Environ. 40 (2006) 3490-3502.

[18]Eugene Yee, Probability Theory as Logic: Data Assimilation for Multiple Source Reconstruction, Pure Appl. Geophys. 169 (2012) 499-517.

[19]Vukovic V, Srebric J, Application of neural networks trained with multizone models for fast detection of contaminant source position in buildings,,ASHRAE Trans. 113 (2007) 154-162.

[20]Bastani A, Haghighat F, Kozinski JA, Contaminant source identification within a building: Toward design of immune buildings,Build. and Environ. 51 (2012) 320-329.

[21]Cai H, Li X, Kong L, Shao X, An optimization method of sensor layout to improve source identification accuracy in the indoor environment, Int. J. of Vent. 11 (2012) 155170.

[22]Hao Cai, Xianting Li, Zhilong Chen, Lingjuan Kong, Fast identification of multiple indoor constant contaminant sources by ideal sensors: A theoretical model and numerical validation, Indoor and Built Environ. 22;6 (2013) 897-909.

[23]Zhang T. F. and Chen Q. Identification of contaminant sources in enclosed spaces by a single sensor. Indoor Air. 17;6 (2007) 439-449.

[24]Li H. Identification of particulate contaminant sources in enclosed space with inverse CFD modeling. Ph.D. Thesis, Dalian University of Technology (in Chinese).2010.

[25]Yin S. Quantitatively identify unsteady gas pollutant release in indoor environment by inverse CFD modeling. Ph.D. Thesis, Dalian University of Technology (in Chinese).2011.

[26]Tengfei (Tim) Zhang, Hongzhu Li, Shugang Wang, Inversely tracking indoor airborne particles to locate their release sources, Atmos. Environ. 55 (2012) 328-338.

[27]Tengfei (Tim) Zhang, Shi Yin, Shugang Wang, An inverse method based on CFD to quantify the temporal release rate of a continuously released pollutant source, Atmos. Environ. 77 (2013) 62-77.

[28]Tengfei (Tim) Zhang, Hongbiao Zhou, Shugang Wang, Inverse identification of the release location, temporal rates and sensor alarming time of an airborne pollutant source, Indoor Air. 25 (2015) 415-427.

[29]Zhiqiang (John) Zhai, Xiang Liu, Haidong Wang, Yuguo Li, Junjie Liu, Experimental verification of tracking algorithm for dynamically-releasing single indoor contaminant. Build. Simul. 5 (2012) 5-14. 
[30]Liu X. and Zhai Z. Location identification for indoor instantaneous point contaminant source by probability-based inverse computational fluid dynamics modeling. Indoor Air. $18 ; 1(2008) 2-11$.

[31]Neupauer R. M. and Wilson J. L. Adjoint method for obtaining backward-in-time location and travel time probabilities of a conservative groundwater contaminant. Water Resour. RES. 35(1999) 3389-3398.

[32]Neupauer R. M. and Wilson J. L. Adjoint-derived location and travel time probabilities for a multidimensional groundwater system. Water Resour. Res. 37(2001) 1657-1668.

[33]Chin D.A. and Chittaluru PV.K. Risk management in wellhead protection. J. Of Water Resour. Planing and Manag.-ASCE. 120 (1994) 294-315.

[34]Dagan G. and Nguyen V. A comparison of travel time and concentration approaches to modeling transport by groundwater. J. Of Comtan. Hydrol. 4 (1989) 79-91.

[35]Jury W. A. and Roth K. Transfer functions and solute movement through soil: theory and applications. J. Of Environ. Qual. 21;4 (1990) 172-173.

[36]Sykes J. F., Wilson J. L. and Andrews R. W. Sensitivity analysis for steady state groundwater flow using adjoint operators. Water Resour. Res. 21;3 (1985) 359-371.

[37]Neupauer R. M. Receptor-based modeling of groundwater contamination. 2000; New Mexico Institute of Mining and Technology.

[38]Lin R. Identification of groundwater contamination sources using probabilities conditioned on measured concentrations. Ph.D. Thesis, University of Virginia. 2003.

[39]Neuparer R.M. and Wilson J.L. Backward probabilistic model of groundwater contaminant in non-uniform and transient flow. Adv. In Water Resour. 25 (2001) 736-746

[40]Launder B. E. and Spalding D. B. The numerical computation of turbulent flows. Comput. Method Appl. Mech. And Eng. 3;2 (1974) 269-289.

[41] Li J. and Liu J. Experimental study of transient air distribution of a jet collision region in an aircraft cabin mock-up. Energy and Build. 127 (2016) 786-793

[42]Liu, W. and Chen Q. Current studies on air distributions in commercial airliner cabins. Theor. And Appl. Mech. Lett. 3;6 (2013) 1-5.

[43]Bianco, V., et al. Numerical investigation of transient thermal and fluid dynamic fields in an executive aircraft cabin. Appl. Therm. Eng. 29;16 (2009) 3418-3425.

[44]Li J. and Liu J. Global airflow field distribution in a cabin mock-up measured via largescale 2D-PIV. Build. Environ. 93 (2015) 234-244.

[45]Chen YL, Jin W. Sensor system design for building indoor air protection. Build. Environ. 43 (2005) 1278-1285.

[46]P Sreedharan, MD Sohn, WW Nazaroff, AJ Gadgil. Influence of indoor transport and mixing time scales on the performance of sensor systems for characterizing contaminant releases. Atmos. Environ. 41 (2007) 9530 - 9542.

[47]Vladimir Vukovic, Paulo Cesar Tabares-Velasc, Jelena Srebric. Real-time identification of indoor pollutant source positions based on neural network locator of contaminant sources and optimized sensor networks. J. of the Air \& Waste Manag. Assoc. 60 (2010) 1034-1048. 
[48]Liu X. Identification of indoor airborne contaminant sources with probability-based inverse modeling methods. Ph.D. Thesis, University of Colorado. 2008 